\title{
Effects of Functionalized Graphene Nanoplatelets on the Morphology and Properties of Phenolic Resins
}

\author{
Jing Dai, ${ }^{1}$ Chao Peng, ${ }^{2}$ Fuzhong Wang, ${ }^{1}$ Guangwu Zhang, ${ }^{1}$ and Zhixiong Huang ${ }^{1}$ \\ ${ }^{1}$ Key Laboratory of Advanced Technology for Special Functional Materials of Ministry of Education, \\ Wuhan University of Technology, Wuhan 430070, China \\ ${ }^{2}$ Faculty of Engineering, China University of Geosciences, Wuhan 430074, China
}

Correspondence should be addressed to Chao Peng; pengchao@cug.edu.cn

Received 13 April 2016; Accepted 3 August 2016

Academic Editor: Alessandro Pegoretti

Copyright ( 92016 Jing Dai et al. This is an open access article distributed under the Creative Commons Attribution License, which permits unrestricted use, distribution, and reproduction in any medium, provided the original work is properly cited.

\begin{abstract}
Graphene nanoplatelets (Gnps) were covalently functionalized by 3-aminopropyltriethoxysilane (KH550) and noncovalently functionalized by Triton X-100, respectively. The morphology and structure of KH550 modified graphene (K-Gnp) and Triton X-100 modified graphene (T-Gnp) were characterized by Fourier transform infrared spectroscopy, scanning electron micrograph, and Raman spectrometer. The influences of K-Gnp and T-Gnp on thermal conductivity, fracture toughness, and thermal stability of the boron phenolic resin (BPR) were investigated. Both covalently functionalized K-Gnp and noncovalently functionalized TGnp not only improve the dispersion of Gnp in the polymer matrix but also increase interfacial bonding strength between the BPR matrix and Gnp, thus leading to the enhanced mechanical property and thermal stability of nanocomposites. Besides this, mechanical property and thermal stability of the BPR containing K-Gnp are superior to those of BPR containing T-Gnp.
\end{abstract}

\section{Introduction}

Phenolic resin (PR) is usually used as a resin matrix for ablative composites because of its excellent thermal stability, mechanical strength, and dielectric properties $[1,2]$. Due to the introduction of boric acid (BA), boron phenolic resin (BPR) possesses enhanced thermal stability, especially the high thermal decomposition temperature and charring yield [3]. BPR and PR are both important resin matrixes of ablation resistant material for thermal protection system (TPS). In order to satisfy the requirements of the ablative materials, most of researchers have focused on incorporating carbon materials into PR. These carbon materials are including carbon nanofibers, carbon nanotubes, and graphite [4-6]. However, there were relatively few reports about the effect of carbon materials on the properties of BPR.

In recent years, carbon nanomaterials have attracted considerable attention from many researchers because of the excellent performance, low density, low cost, and a large number of potential applications. Graphene [7-12] has been widely used as an ideal filler for polymers, due to its unique physical property, high specific area, and relatively low price. Many researchers have selected Gnp/graphene as a novel filler for polymer composites. Wang et al. [13] investigated the factors of Gnp sizes and dispersion on the mechanical and thermal properties of epoxy nanocomposites. The result showed that larger nanoplatelets (Gnp-5) not only exhibit greater reinforcement of the composites modulus than Gnp-C750 but also improve thermal conductivity of epoxy more effectively. Chandrasekaran et al. [14] studied the effect of Gnp on the electrical and thermal conductivity, fracture toughness, and storage modulus of the nanocomposite. The results revealed that the Gnp is an effective reinforcement of epoxies for their mechanical properties. Liu et al. [15] investigated the effect of graphene nanosheets on morphology, thermal stability, and flame retardancy of epoxy resin (ER) and found that graphene nanosheets change the decomposition pathway of ER at a high temperature, enhance the thermal stability, and promote the formation of carbon residue. Chatterjee et al. [16] studied the influence of Gnps and carbon nanotubes (CNTs) on the mechanical properties of epoxybased nanocomposites and the results indicated that the 
nanocomposites containing Gnps have superior tensile and compressive strength compared to nanocomposite containing CNTs.

Due to large surface area and strong van der Waals force, graphene tends to form irreversible agglomerates in polymer matrix and uniform dispersion of Gnp in the polymer matrix. In order to ensure a good dispersion of Gnp in the polymer matrix, functionalization process of Gnp is conducted. Two approaches, including covalent functionalization and noncovalent modification with various organic molecules, are usually used for modifying Gnp surface. Covalent functionalization can be achieved by yielding covalent linkages at the Gnp-polymer interface through the reaction between the carboxyl or hydroxyl groups of Gnp surface and surfactants' groups. Lee et al. [17] prepared silanefunctionalized graphene oxides (Go) with four different selfassembled monolayers and found that functionalized Go can strengthen the interfacial bonding between the carbon fibers and epoxy adhesive. Ma et al. [18] covalently modified graphene platelets by the reaction of the Gnps' epoxide groups and the end-amine groups of a commercial longchain surfactant and found that the modified Gnps (m-Gnps) increase Young's modulus, fracture energy release rate, and glass transition temperature of epoxy by $14 \%, 387 \%$, and $13 \%$, respectively. In addition, noncovalent functionalization can be achieved by polymer wrapping, adsorption of surfactants, or small molecules via p-p stacking interactions. Li et al. [19] noncovalently functionalized graphene with poly(sodium 4-styrenesulfonate) (PSS) and found that functionalization process improves interfacial bonding between matrix and graphene. Teng et al. [20] noncovalently functionalized graphene nanosheets (GNS) by a functional segmented polymer chain (Py-PGMA) and found that thermal conductivity of Py-PGMA-GNS/epoxy composites increases remarkably owing to the well dispersion of GNS and interfacial interaction between GNS and resin. Although polymers modified by Gnp have been investigated by many researchers, the application of Gnp in BPR was rarely reported previously.

In this study, Gnp/BPR composite was prepared by adding functionalized Gnp to BPR resin. Firstly, we modified the surface of Gnp to obtain functionalized Gnp by two different approaches. The first one is covalent functionalization method of bonding 3-Triethoxysilylpropylamine (KH550) coupling agent containing epoxy ended groups on the surface of the Gnp. The second one is noncovalent functionalization method of attaching electrostatic repulsion of the hydrophilic group of nonionic organic surfactant Triton X-100 to the surface of Gnp. Then we mixed modified Gnp with BPR resin and studied the effect of functionalization Gnp on the thermal stability and mechanical properties of BPR.

\section{Experimental}

2.1. Materials. Gnp was purchased from XG Sciences (Lansing, Michigan, USA). The average diameter of Gnp is approximately $5 \mathrm{~mm}$ and the thickness of Gnps is less than $10 \mathrm{~nm}$. BPR was purchased from Benbu-Temperature Resistant Resin Factor Co., Ltd. (Anhui, China). 3-Triethoxysilylpropylamine (KH550) was produced by Nabo New Material
Technology Co., Ltd., (Tianjin, China). Triton X-100, ethanol, acetone, and methanol were all made from Sinopharm Chemical Reagent Co., Ltd. (Shanghai, China).

2.2. KH550 Modification of Gnp. $0.4 \mathrm{~g}$ of Gnp was dispersed in $400 \mathrm{~mL}$ ethanol, followed by the addition of $2.5 \mathrm{~g}$ of $\mathrm{KH} 550$ and $0.5 \mathrm{~mL} \mathrm{H}_{2} \mathrm{O}$ to the solution. Then, the mixed solution was placed in an oil bath and stirred at $70^{\circ} \mathrm{C}$ for $12 \mathrm{~h}$. KH550 functionalized Gnp (K-Gnp) was filtered off and washed several times with ethanol, acetone, and methanol. The residue was dried at $80^{\circ} \mathrm{C}$ in a vacuum oven overnight.

2.3. Triton X-100 Modification of Gnp. Pristine Gnp powder $(0.4 \mathrm{~g})$ and Triton X-100 (0.6 g) were added into $400 \mathrm{~mL}$ ethanol with $1 \mathrm{~h}$ of ultrasonication. Then, the solution was placed in an oil bath and stirred at $70^{\circ} \mathrm{C}$ for $12 \mathrm{~h}$. The resulting Triton-functionalized Gnp (T-Gnp) has undergone the same steps with Section 2.2.

2.4. Preparation of $G n p / B P R$ Composite. BPR composites containing as-produced Gnp, T-Gnp, and K-Gnp were prepared by the following procedures. $40 \mathrm{~g}$ of BPR was added to the $40 \mathrm{~mL}$ ethanol and then the mixed solution was placed in an oil bath and stirred at $70^{\circ} \mathrm{C}$ until the BPR was dissolved in ethanol. Then, the modified Gnp was dispersed in the BPR solution with a bath sonication for $1 \mathrm{~h}$ at room temperature to prepare well dispersed Gnp/BPR. After bath sonication, the mixture was placed in an oil bath and stirred at $70^{\circ} \mathrm{C}$ for $12 \mathrm{~h}$ until the ethanol was almost evaporated. The product was dried in an oven at $60^{\circ} \mathrm{C}$ under the vacuum pressure of $0.085 \mathrm{MPa}$ till a constant weight.

Subsequently, the resulting mixture was degassed at $65^{\circ} \mathrm{C}$ to remove air bubbles and any residual solvent, followed by a casting procedure in silicone molds. Finally, the products were cured at $80^{\circ} \mathrm{C}, 100^{\circ} \mathrm{C}, 120^{\circ} \mathrm{C}, 150^{\circ} \mathrm{C}$, and $150^{\circ} \mathrm{C}$ for $5 \mathrm{~h}$, $2 \mathrm{~h}, 2 \mathrm{~h}$, and $1 \mathrm{~h}$ each. The neat BPR was also prepared by the above procedures. The composites containing as-produced Gnp, K-Gnp, and T-Gnp were referred to as P-Gnp/BPR, KGnp/BPR, and T-Gnp/BPR, respectively.

\section{Measurements}

The surfactant group of Gnp was characterized by Nicolet FTIR spectrophotometer (Nicolet 6700, Nicolet Instrument Company, USA). The wave numbers of spectra were recorded from 500 to $4000 \mathrm{~cm}^{-1}$ at a resolution of $2 \mathrm{~cm}^{-1}$ using $\mathrm{KBr}$ pellets.

The tensile fracture surfaces of BPR-based samples and the microstructures of P-Gnp, K-Gnp, and T-Gnp were characterized by Quanta FEG450 environmental scanning electron microscope at an accelerated voltage of $15 \mathrm{kV}$. The sheets were dispersed in ethanol by sonication for $30 \mathrm{~min}$ and then were gold coated ( $3 \mathrm{~nm}$ thick gold) to avoid charging prior to observation.

Raman spectra were recorded on a SENTERRA Micro Raman Spectroscopy (Bruker Instrument, Germany) with $500 \mathrm{~nm}$ laser excitation. 


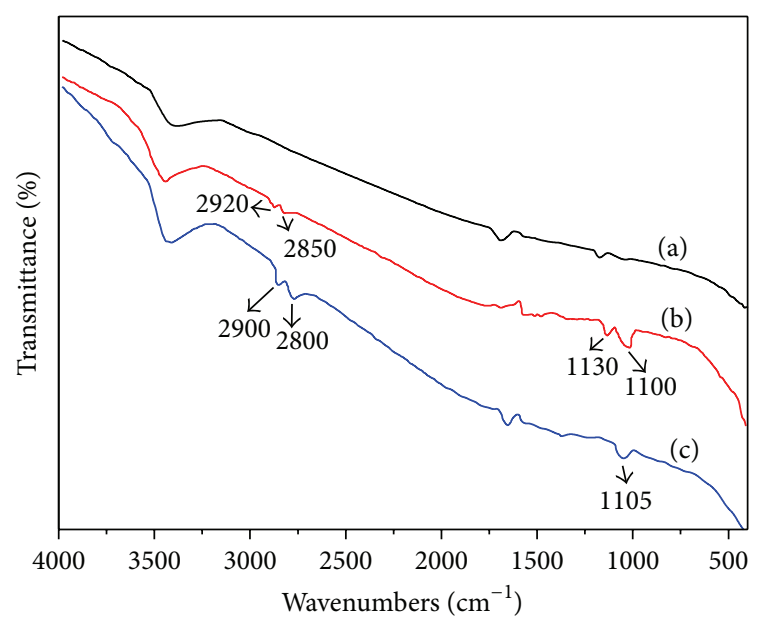
(a) P-Gnp
(b) K-Gnp
(c) T-Gnp

Figure 1: FTIR spectra of (a) P-Gnp, (b) K-Gnp, and (c) T-Gnp.

Three-point bending flexural tests were conducted according to GB/T 2567-2008 method at room temperature [13] using a universal testing machine (RGM-4100, maximum capacity of $100 \mathrm{kN}$, China). The tests were performed at a constant loading speed of $2 \mathrm{~mm} \mathrm{~min}^{-1}$. The dumbbell-shaped samples were prepared with dimension of $90 \mathrm{~mm} \times 12.7 \mathrm{~mm}$ $\times 3 \mathrm{~mm}$. At least five specimens were tested for each group and all the results were calculated based on the average value of five tests.

The thermal conductivities of the neat BPR and BPR composites at room temperature were measured by TC7000 HLaser flash apparatus at room temperature. The samples were cut into square shape with length of $8 \mathrm{~mm}$ and thickness of $1.6 \mathrm{~mm}$.

The thermal behavior of nanocomposite was characterized by TGA, which was conducted by synchronous thermal analysis (NETZSCH STA 449 F3 typed thermal analyzer, Germany) at a heating rate of $20^{\circ} \mathrm{C} \cdot \mathrm{min}^{-1}$ with Nitrogen flow rates of $50 \mathrm{~mL} \mathrm{~min}^{-1}$.

\section{Results and Discussion}

4.1. Characterization of Gnp. Figure 1(a) shows the FT-IR spectroscopy results of P-Gnp, K-Gnp, and T-Gnp. The peaks located at $3450 \mathrm{~cm}^{-1}, 1705 \mathrm{~cm}^{-1}$, and $1240 \mathrm{~cm}^{-1}$ in the Gnps spectrum were attributed to the typical vibrations of $-\mathrm{OH}$, $\mathrm{C}=\mathrm{O}$, and $\mathrm{C}-\mathrm{O}$ bonds, respectively. It indicates that the hydroxyl and carboxyl groups are on the surface of P-Gnp. In the spectra of the K-Gnp (as is shown in Figure 1(b)), the peaks appearing at $2920 \mathrm{~cm}^{-1}$ and $2850 \mathrm{~cm}^{-1}$ correspond to symmetric and asymmetric vibrations of alkyl groups, which are caused by the silane coupling agent of the K-Gnp. Moreover, the appearance of the peaks at $1125 \mathrm{~cm}^{-1}$ and $1045 \mathrm{~cm}^{-1}$ in the spectra of the K-Gnp indicates the formation of Si-O$\mathrm{C}$ and $\mathrm{Si}-\mathrm{O}-\mathrm{Si}$, which is a further evidence of the presence of silane coupling agent on the surface of Gnp. In the spectrum

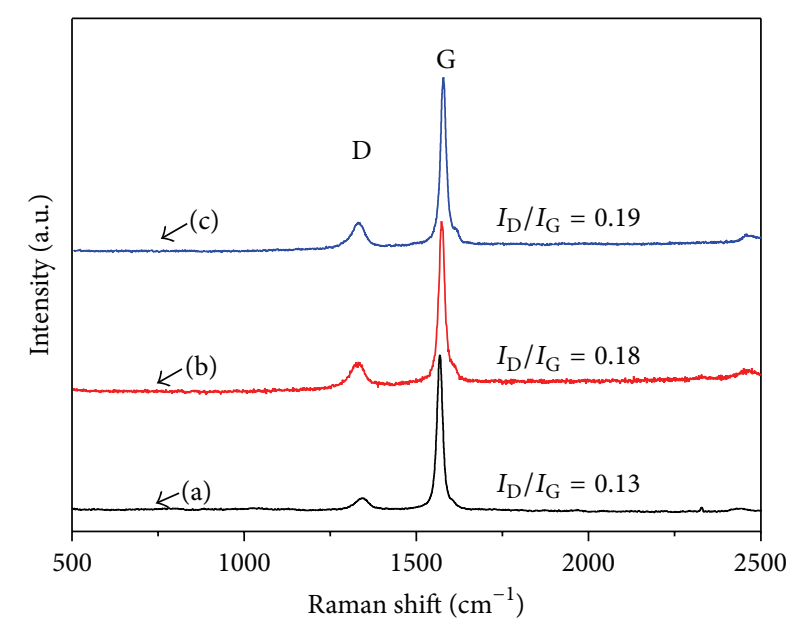
(a) P-Gnp
(b) K-Gnp
(c) T-Gnp

Figure 2: Raman spectra of (a) P-Gnp, (b) K-Gnp, and (c) T-Gnp.

of T-Gnp (as is shown in Figure 1(c)), the peaks at $1105 \mathrm{~cm}^{-1}$ correspond to $\mathrm{C}-\mathrm{O}$ stretching bands. The absorption peaks at $2800 \mathrm{~cm}^{-1}$ and $2900 \mathrm{~cm}^{-1}$ are probably attributed to the symmetric and asymmetric stretching vibration of $-\mathrm{CH}_{3}$ and $-\mathrm{CH}_{2}$ groups from Triton X-100, respectively. It indicated that the Triton X-100 molecules have been adsorbed on the surface of the Gnp.

Raman spectra for the P-Gnp, K-Gnp, and T-Gnp samples are shown in Figure 2. For P-Gnp, K-Gnp, and T-Gnp, two characteristic peaks were observed at $1340 \mathrm{~cm}^{-1}$ (D band) and $1570 \mathrm{~cm}^{-1}$ ( $\mathrm{G}$ band). $\mathrm{G}$ band originates primarily from the presence of $\mathrm{sp} 2$ carbon network, while the $\mathrm{D}$ band arises from defects [21]. The intensity ratio $\left(I_{\mathrm{D}} / I_{\mathrm{G}}\right)$ of the $\mathrm{D}$ band to $\mathrm{G}$ band corresponded to the defect level of the material. The larger the $I_{\mathrm{D}} / I_{\mathrm{G}}$ ratio is, the higher the defect level of the material is. In this study, we found that the $D$ band to $G$ band ratio of K-Gnp $\left(I_{\mathrm{D}} / I_{\mathrm{G}}=0.18\right)$ and T-Gnp $\left(I_{\mathrm{D}} / I_{\mathrm{G}}=0.19\right)$ is higher than that of $\mathrm{P}-\mathrm{Gnp}\left(I_{\mathrm{D}} / I_{\mathrm{G}}=0.13\right)$. The result implied that the defect level of these functionalized Gnps is higher than the as-produced Gnp. It further indicated that Gnp has been modified successfully by the KH550 and the Triton X100.

4.2. Dispersion of Gnp. Due to the strong $\mathrm{p}-\mathrm{p}$ interactions, Gnp has an inherent tendency to form agglomerates, resulting in an inhomogeneous dispersion of nanofillers in polymer matrix [16]. Figure 3 shows that the smooth surface appears in the pristine Gnp, while rough surfaces can be observed in K-Gnp and T-Gnp. In addition, we can find some white dots on the surface of K-Gnp and T-Gnp. These morphologies implied that K-Gnp and T-Gnp have been successfully functionalized by KH550 and Triton X-100, respectively.

Figures 4(a)-4(c) show the dispersion state of P-Gnp, K-Gnp, and T-Gnp in ethanol. We found that P-Gnp (Figure 4(b)) precipitated in ethanol in a relatively short time (1-6h), while K-Gnp (Figure 4(b)) and T-Gnp (Figure 4(c)) 


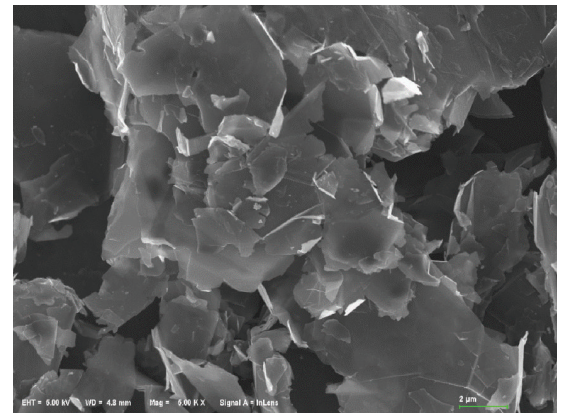

(a)

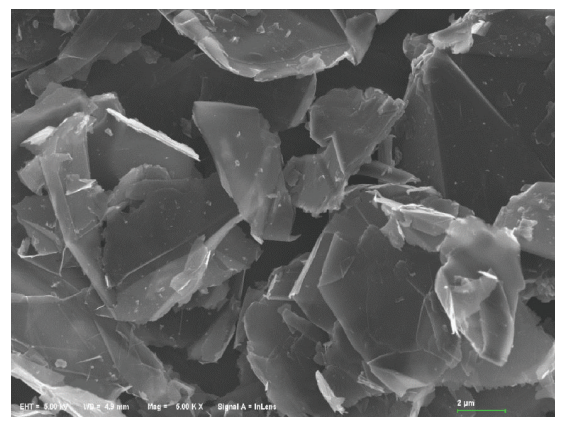

(b)

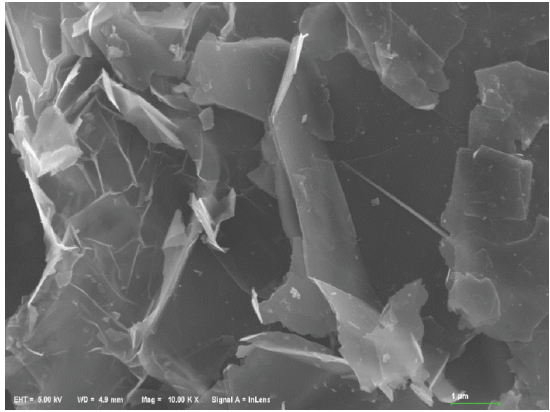

(c)

FIgURE 3: Scanning electron micrographs of (a) P-Gnp, (b) K-Gnp, and (c) T-Gnp.

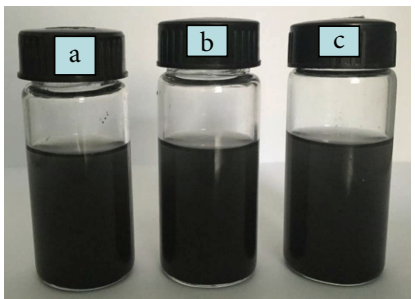

$1 \mathrm{~h}$

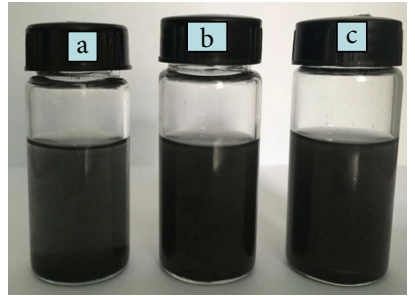

$6 \mathrm{~h}$

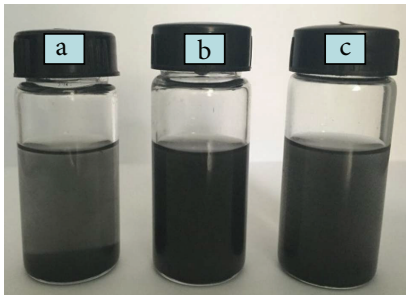

$10 \mathrm{~h}$

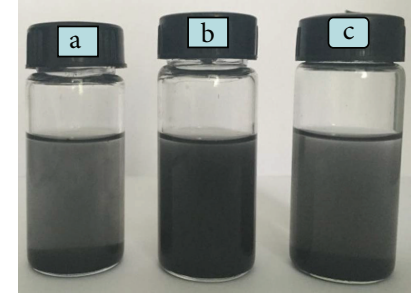

$24 \mathrm{~h}$

Figure 4: Dispersion states of (a) P-Gnp, (b) K-Gnp, and (c) T-Gnp in ethanol solvents.

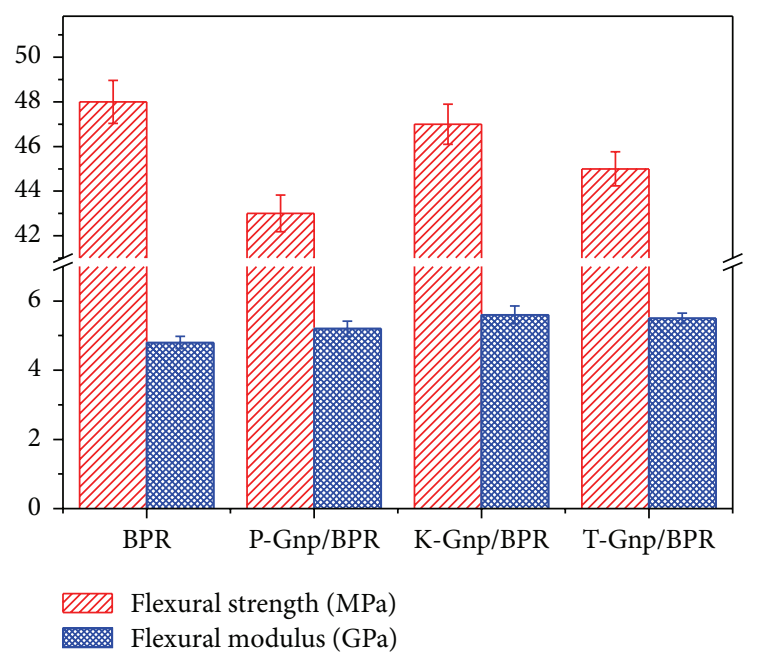

FIgURE 5: Flexural properties of neat BPR and its composites.

still remained soluble in ethanol. After $10 \mathrm{~h}$, a clear solidliquid interface appeared in the P-Gnp specimen, while TGnp tended to separate from the ethanol solvent. After $24 \mathrm{~h}$, the separation process of T-Gnp was completed and K-Gnp still remained soluble. This observation proved that K-Gnp and T-Gnp both remain insoluble in ethanol.

4.3. Mechanical Results. The effects of Gnp on the flexural strength and modulus of BPR resin were investigated by the flexural tests. Figure 5 presents flexural properties of the neat BPR, P-Gnp/BPR, K-Gnp/BPR, and T-Gnp/BPR.
The flexural moduli of P-Gnp/BPR, K-Gnp/BPR, and T-Gnp/BPR increased by $8.3 \%, 16.6 \%$, and $14.5 \%$ in comparison with that of neat BPR. This is because Gnp with high aspect ratio and moduli can undertake more load to protect the resin matrix. The K-Gnp and T-Gnp showed a greater increase of the flexural moduli than the P-Gnp owing to the improved interfacial interactions and good dispersion.

In contrast, the addition of P-Gnp decreased the flexural strength of BPR resin by $10.4 \%$. This is because of the weak interfacial interaction between the BPR resins and P-Gnp, which is shown in Figure 4(a). Some reports also showed that the addition of Gnp leads to a decrease in mechanical strength of epoxy matrix [22]. Besides this, the weak adhesion between resin matrix and the outer layers of Gnp, as well as the weak Gnp interlayer forces (van der Waals forces), results in a poor mechanical strength [23]. However, the composites with KGnp and T-Gnp showed a less reduction in flexural strength than the unmodified Gnp. As is shown in Figure 4(b), the surface of covalent functionalized K-Gnp with many oxygen functional groups can be dispersed well in resin. The strong interfacial adhesion facilitates efficient load transfer from matrix to fillers and consequently enhances the reinforcing effect. In addition, compared with P-Gnp, T-Gnp also has a stronger interaction with polymer matrix (which is shown in Figure 4(c)).

4.4. Morphologies. Figure 6(a) shows the extraction of PGnp (see the white arrow) and gap between P-Gnp and $\mathrm{BPR}$ resin (the white dotted line); these phenomena are corresponding to the weak interfacial bonding between $\mathrm{P}$ Gnp and BPR resin. Figure 6(b) shows that K-Gnp is well embedded in the BPR resin (the black arrows) and this is 


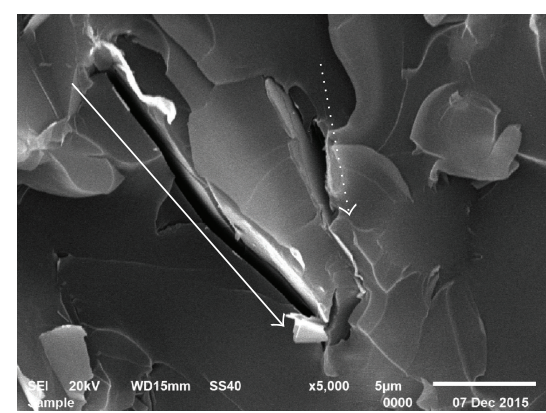

(a)

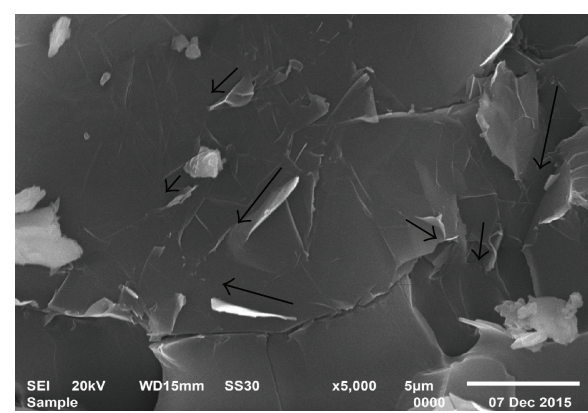

(b)

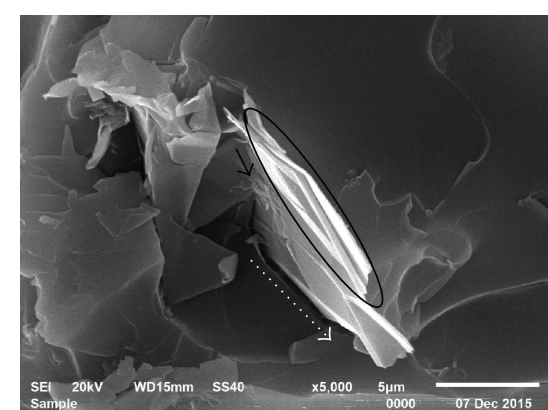

(c)

FIGURE 6: Scanning electron micrographs of fracture surfaces of (a) P-Gnp/BPR, (b) K-Gnp/BPR, and (c) T-Gnp/BPR composites.

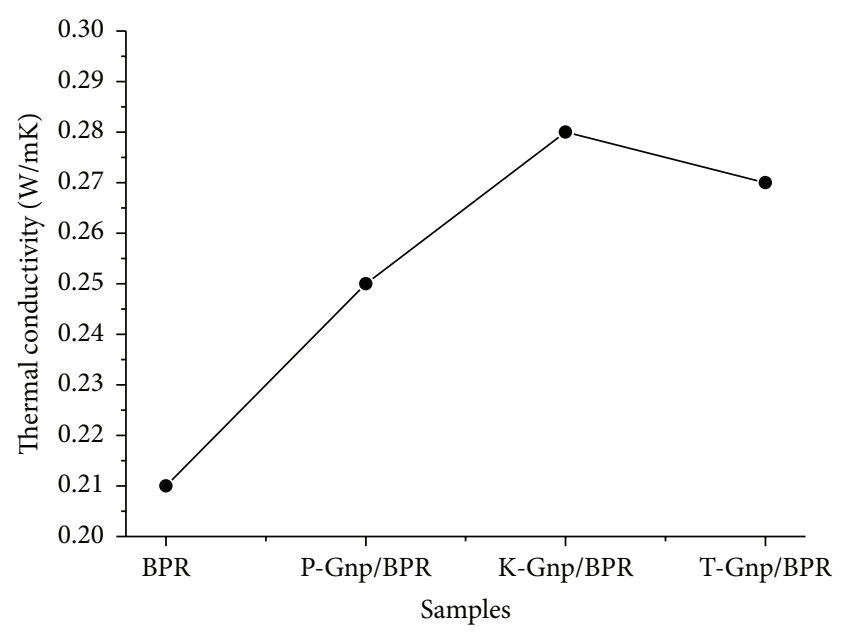

FIGURE 7: Thermal conductivities of neat BPR and its composites.

due to the abundant availability of oxygen groups on K-Gnp. Once the load exceeds the filler/matrix interfacial strength, the K-Gnp would be pulled out from the BPR resin. However, the obvious space between K-Gnp and BPR resin cannot be found, because of the strong interfacial interaction between K-Gnp and BPR resin. In Figure 6(c), we can see that a three-layer graphene which is attributed to a thin Gnp was peeled from the thicker one during the sudden breakage of the composite [24]. In addition, part of T-Gnp is combined closely with resin (see the black arrow) and a small space between the T-Gnp and BPR resin (see the white dotted line) proved that it is difficult to pull out the Gnp from resin. The above phenomena indicate that K-Gnp has stronger interfacial interaction with resin than primary Gnp and TGnp.

4.5. Thermal Properties of Gnp-Based Composites. Thermal conductivities of BPR resin, P-Gnp/BPR, K-Gnp/BPR, and T-Gnp/BPR composites are shown in Figure 7. The thermal conductivities of the Gnp/BPR nanocomposites improved as the addition of $\mathrm{P}-\mathrm{Gnp}$ in polymer matrix increased from 0.21 to 0.25 . This is because the Gnp with high intrinsic thermal conductivity is beneficial for the plenty formation of thermally conductive pathways in the resin matrix. In
TABLE 1: Thermal characteristics of the neat PBR and its composites.

\begin{tabular}{lccc}
\hline Samples & ${ }^{\mathrm{a}} T_{10}\left({ }^{\circ} \mathrm{C}\right)$ & ${ }^{\mathrm{b}} T_{\max }\left({ }^{\circ} \mathrm{C}\right)$ & ${ }^{\mathrm{c}} R_{1000}\left({ }^{\circ} \mathrm{C}\right)(\%)$ \\
\hline BPR & 320 & 535 & 62.5 \\
P-Gnp/BPR & 321 & 585 & 66.2 \\
K-Gnp/BPR & 430 & 596 & 70.1 \\
T-Gnp/BPR & 420 & 590 & 68.2 \\
\hline
\end{tabular}

${ }^{a}$ Thermal decomposition temperature at $10 \%$ weight loss.

${ }^{\mathrm{b}}$ The temperature of the maximum degradation rate.

${ }^{\mathrm{c}}$ Residue weight at $1000^{\circ} \mathrm{C}$.

addition, the thermal conductivities of BPR with K-Gnp and T-Gnp increased slightly from 0.25 to 0.28 and from 0.25 to 0.27 , respectively. The superior performance of $\mathrm{K}$ Gnp and T-Gnp on the thermal conductivities of resins is related to the following reasons. First, the good dispersion of functionalized Gnp in the resin matrix reduces the average free path length of heat transfer, which is mainly attributed to the transport of phonons. That means the better dispersion can result in the greater thermal conductivity. Second, the functionalized Gnp enhances the interaction between Gnp and matrix and reduces barriers of phonon transport, leading to a high thermal conductivity.

4.6. Thermal Properties of Gnp-Based Composites. The thermal properties of BPR and Gnp/BPR composites were evaluated by thermogravimetric analysis (TGA) under nitrogen atmosphere and the results are shown in Figure 8 and Table 1. The thermal resistance of BPR resin was obviously improved by incorporating $1 \mathrm{wt} \%$ of Gnp. The thermal decomposition temperatures $\left(T_{10}\right)$ defined as the temperature at $10 \%$ weight loss and residual weights of the samples at $1000^{\circ} \mathrm{C}$ are shown in Table 1 . We can see that $T_{10}$ of P-Gnp/BPR, K$\mathrm{Gnp} / \mathrm{BPR}$, and T-Gnp/BPR composites increase to $1^{\circ} \mathrm{C}, 100^{\circ} \mathrm{C}$, and $110^{\circ} \mathrm{C}$, respectively. It is obvious that the addition of functionalized Gnp leads to a remarkable increase in BPR thermal stability. The significant effect of functionalized Gnp is mainly attributed to the good matrix-Gnp interaction and thermal conductivity as well as their barrier effect. With the addition of Gnp, the average residue yields of resin at $1000^{\circ} \mathrm{C}$ of P-Gnp/BPR, K-Gnp/BPR, and T-Gnp/BPR increased from $62.5 \%$ to $66.2 \%, 70.1 \%$, and $68.2 \%$ respectively. From the DTG curve (Figure 8), we can see that the maximum heat 

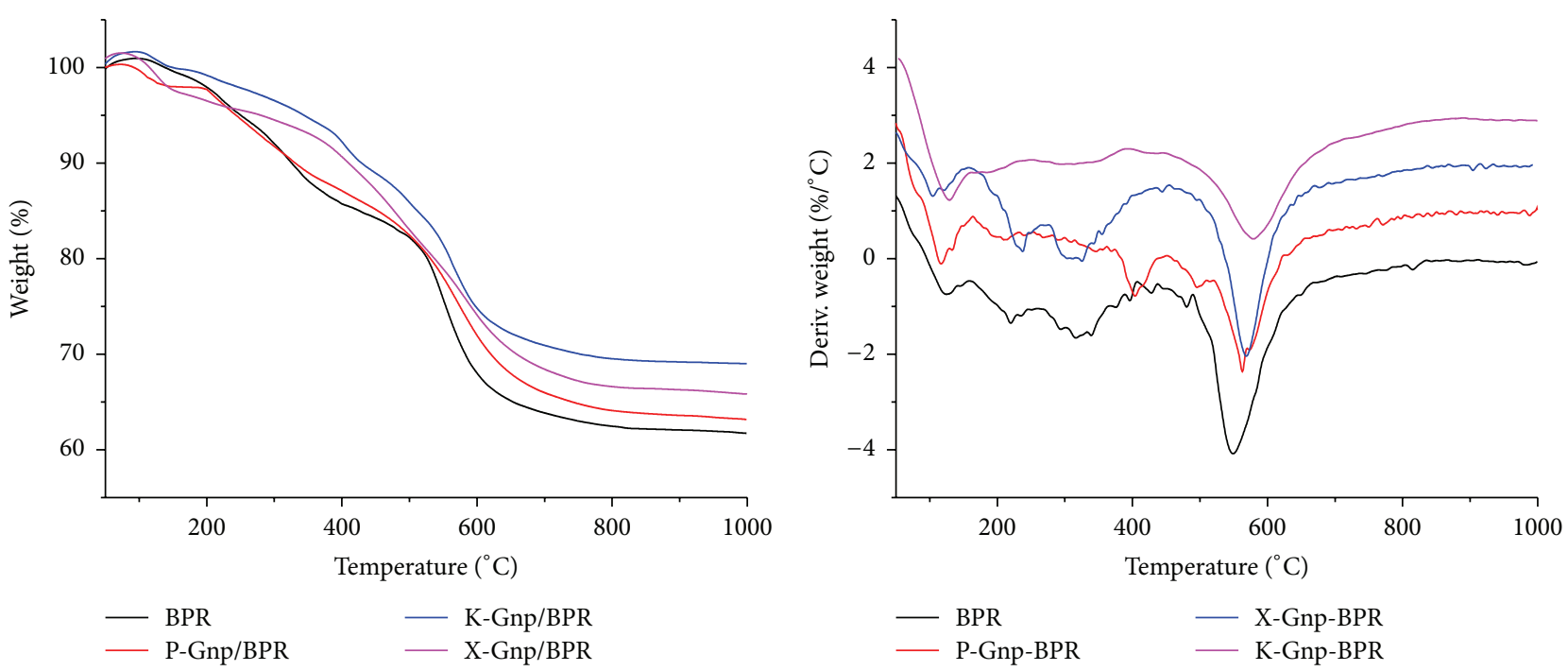

FIGURE 8: DTG curves of the neat BPR and its composites in $\mathrm{N}_{2}$ atmosphere.

loss rates of BPR, P-Gnp/BPR, K-Gnp/BPR, and T-Gnp/BPR composites are $535^{\circ} \mathrm{C}, 585^{\circ} \mathrm{C}, 596^{\circ} \mathrm{C}$, and $590^{\circ} \mathrm{C}$ respectively. P-Gnp/BPR, K-Gnp/BPR, and T-Gnp/BPR increased the maximum heat loss rates by $13.7^{\circ} \mathrm{C}, 19.5^{\circ} \mathrm{C}$, and $29.7^{\circ} \mathrm{C}$ in comparison with neat BPR. From the above analysis, we can conclude that KH550 can improve the thermal stability of BPR more effectively than Triton X-100.

The thermal properties of Gnp/BPR composites can be also attributed to the formation of graphitized carbon. Yum et al. [25] revealed that the graphitic structures formed around the CNTs improves the high temperature ablation resistance of composites. Si et al. [21] reported that the graphite oxide (GO) promotes PR to form a stable graphite structure. Yi et al. [26] found that GO is useful to accelerate the graphitization of furan resin carbon. In this study, graphitization of BPR can be achieved by adding functionalized Gnp. The Raman spectra and XRD analysis were used to examine the BPR carbon and Gnp/BPR carbon, which are prepared by treating BPR and $\mathrm{Gnp} / \mathrm{BPR}$ composites at $1000^{\circ} \mathrm{C}$ under $\mathrm{N}_{2}$ atmosphere for $2 \mathrm{~h}$. $I_{\mathrm{D}} / I_{\mathrm{G}}$ ratios of BPR carbon, Gnp/BPR carbon, KGnp/BPR carbon, and T-Gnp/BPR carbo nare 2.13, 1.98, 1.87, and 1.90, respectively (Figure 9), indicating that Gnp can promote graphitization of Gnp/BPR composites and can further improve the content of graphitization carbon in the carbon layer. In addition, it is notable that the Raman scattering intensities of Gnp/BPR carbon, K-Gnp/BPR carbon, and T$\mathrm{Gnp} / \mathrm{BPR}$ carbon are very close. It implies that the improved graphitization degree of carbon layer is mainly owing to the formation of graphene structure. The higher the degree of graphitization is, the more dense the carbon layer is. It can also lead to a more obvious barrier effect of the exchange between the matter and energy by material and the flame.

\section{Conclusions}

In this study, covalently functionalized K-Gnp and noncovalently functionalized T-Gnp were successfully prepared and

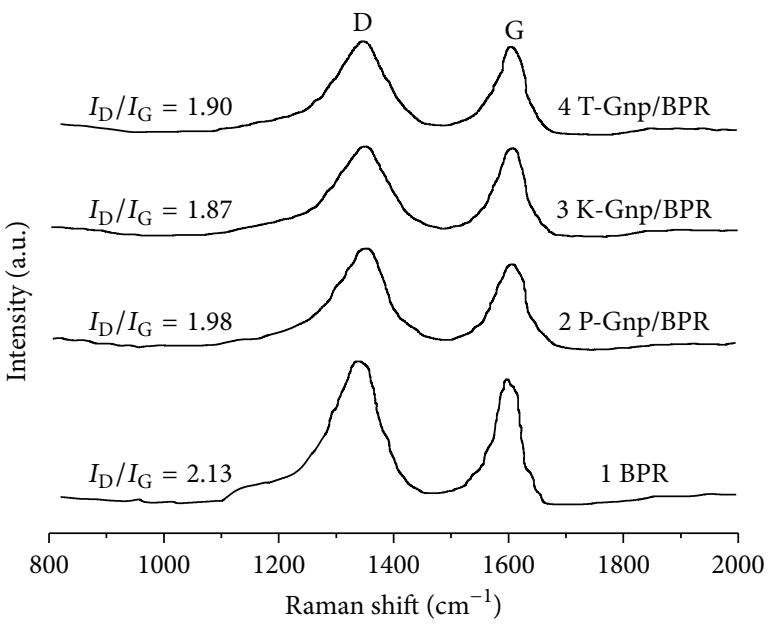

FIGURE 9: Raman spectra of carbon of BPR and its composites.

the effects of P-Gnp, K-Gnp, and T-Gnp on BPR on mechanical properties, thermal conductivity, and thermal properties of BPR-based composites were investigated. It demonstrated that K-Gnp and T-Gnp both have a better interfacial adhesion with BPR which is resulting from the covalently functionalization by KH550 and noncovalently functionalization by Triton X-100. K-Gnp/BPR and T-Gnp/BPR have better mechanical and thermal properties than Gnp/BPR. This improvement of K-Gnp and T-Gnp is ascribed to the successful functionalization of Gnp. The enhanced interfacial adhesion between functionalized Gnp and epoxy matrix can be verified from the IR/Raman spectra and SEM photos. The mechanical property measurements disclosed that K-Gnp and T-Gnp exhibit greater reinforcement in the modulus of the composites than P-Gnp, while the composites with K-Gnp and T-Gnp show a less reduction in strength than the P-Gnp. In addition, thermal conductivities, the average residue yield, 
and graphitization degree of carbon layer of $\mathrm{BPR}$ resin are all increased with the addition of K-Gnp and T-Gnp.

\section{Competing Interests}

The authors declare that there is no conflict of interests regarding the publication of this article.

\section{References}

[1] R. Balaji and M. Sasikumar, "A study on the effect of cenosphere on thermal and ablative behavior of cenosphere loaded ceramic/phenolic composites," Polymer, vol. 55, no. 25, pp. 6634-6639, 2014.

[2] Y. Chen, P. Chen, C. Hong, B. Zhang, and D. Hui, "Improved ablation resistance of carbon-phenolic composites by introducing zirconium diboride particles," Composites Part B: Engineering, vol. 47, no. 3, pp. 320-325, 2013.

[3] Z. He, "Synthesis of phenolic aldehyde resin modified by boron for anti-friction materials use," Guizhou Chemical Industry, vol. 3, pp. 11-12, 1999.

[4] M. Yoonessi, H. Toghiani, R. Wheeler, L. Porcar, S. Kline, and C. U. Pittman Jr., "Neutron scattering, electron microscopy and dynamic mechanical studies of carbon nanofiber/phenolic resin composites," Carbon, vol. 46, no. 4, pp. 577-588, 2008.

[5] S. J. Kim and H. Jang, "Friction and wear of friction materials containing two different phenolic resins reinforced with aramid pulp," Tribology International, vol. 33, no. 7, pp. 477-484, 2000.

[6] E. Bafekrpour, G. P. Simon, C. Yang, M. Chipara, J. Habsuda, and B. Fox, "A novel carbon nanofibre/phenolic nanocomposite coated polymer system for tailoring thermal behaviour," Composites Part A: Applied Science and Manufacturing, vol. 46, no. 1, pp. 80-88, 2013.

[7] C. Lee, X. Wei, J. W. Kysar, and J. Hone, "Measurement of the elastic properties and intrinsic strength of monolayer graphene," Science, vol. 321, no. 5887, pp. 385-388, 2008.

[8] J. Liang, Y. Wang, Y. Huang et al., "Electromagnetic interference shielding of graphene/epoxy composites," Carbon, vol. 47, no. 3 , pp. 922-925, 2009.

[9] H.-B. Zhang, W.-G. Zheng, Q. Yan et al., "Electrically conductive polyethylene terephthalate/graphene nanocomposites prepared by melt compounding," Polymer, vol. 51, no. 5, pp. 11911196, 2010.

[10] K. Kalaitzidou, H. Fukushima, and L. T. Drzal, "Multifunctional polypropylene composites produced by incorporation of exfoliated graphite nanoplatelets," Carbon, vol. 45, no. 7, pp. 14461452, 2007.

[11] L. T. Drzal and H. Fukushima, "Expanded graphite and products produced therefrom," US Patent 8501858B2, 2006.

[12] XG Sciences, http://xgsciences.com/.

[13] F. Wang, L. T. Drzal, Y. Qin, and Z. Huang, "Mechanical properties and thermal conductivity of graphene nanoplatelet/epoxy composites," Journal of Materials Science, vol. 50, no. 3, pp. 10821093, 2015.

[14] S. Chandrasekaran, C. Seidel, and K. Schulte, "Preparation and characterization of graphite nano-platelet (GNP)/epoxy nanocomposite: mechanical, electrical and thermal properties," European Polymer Journal, vol. 49, no. 12, pp. 3878-3888, 2013.
[15] S. Liu, H. Yan, Z. Fang, and H. Wang, "Effect of graphene nanosheets on morphology, thermal stability and flame retardancy of epoxy resin," Composites Science and Technology, vol. 90, pp. 40-47, 2014.

[16] S. Chatterjee, F. Nafezarefi, N. H. Tai, L. Schlagenhauf, F. A. Nüesch, and B. T. T. Chu, "Size and synergy effects of nanofiller hybrids including graphene nanoplatelets and carbon nanotubes in mechanical properties of epoxy composites," Carbon, vol. 50, no. 15, pp. 5380-5386, 2012.

[17] C. Y. Lee, J.-H. Bae, T.-Y. Kim, S.-H. Chang, and S. Y. Kim, "Using silane-functionalized graphene oxides for enhancing the interfacial bonding strength of carbon/epoxy composites," Composites Part A: Applied Science and Manufacturing, vol. 75, pp. 11-17, 2015.

[18] J. Ma, Q. Meng, I. Zaman et al., "Development of polymer composites using modified, high-structural integrity graphene platelets," Composites Science and Technology, vol. 91, no. 2, pp. 82-90, 2014.

[19] Y. Li, J. Tang, L. Huang et al., "Facile preparation, characterization and performance of noncovalently functionalized graphene/epoxy nanocomposites with poly(sodium 4styrenesulfonate)," Composites Part A: Applied Science and Manufacturing, vol. 68, pp. 1-9, 2015.

[20] C.-C. Teng, C.-C. M. Ma, C.-H. Lu et al., "Thermal conductivity and structure of non-covalent functionalized graphene/epoxy composites," Carbon, vol. 49, no. 15, pp. 5107-5116, 2011.

[21] J. Si, J. Li, S. Wang, Y. Li, and X. Jing, "Enhanced thermal resistance of phenolic resin composites at low loading of graphene oxide," Composites Part A: Applied Science and Manufacturing, vol. 54, no. 4, pp. 166-172, 2013.

[22] M. Martin-Gallego, M. M. Bernal, M. Hernandez, R. Verdejo, and M. A. Lopez-Manchado, "Comparison of filler percolation and mechanical properties in graphene and carbon nanotubes filled epoxy nanocomposites," European Polymer Journal, vol. 49, no. 6, pp. 1347-1353, 2013.

[23] F. Wang, L. T. Drzal, Y. Qin, and Z. Huang, "Effects of functionalized graphene nanoplatelets on the morphology and properties of epoxy resins," High Performance Polymers, vol. 28, no. 5, pp. 525-536, 2016.

[24] W.-S. Kuo, N.-H. Tai, and T.-W. Chang, "Deformation and fracture in graphene nanosheets," Composites Part A: Applied Science and Manufacturing, vol. 51, no. 7, pp. 56-61, 2013.

[25] S. H. Yum, S. H. Kim, W. I. Lee, and H. Kim, "Improvement of ablation resistance of phenolic composites reinforced with low concentrations of carbon nanotubes," Composites Science and Technology, vol. 121, pp. 16-24, 2015.

[26] S. Yi, J. Chen, H. Li, L. Liu, X. Xiao, and X. Zhang, "Effect of graphite oxide on graphitization of furan resin carbon," Carbon, vol. 48 , no. 3, pp. 926-928, 2010. 

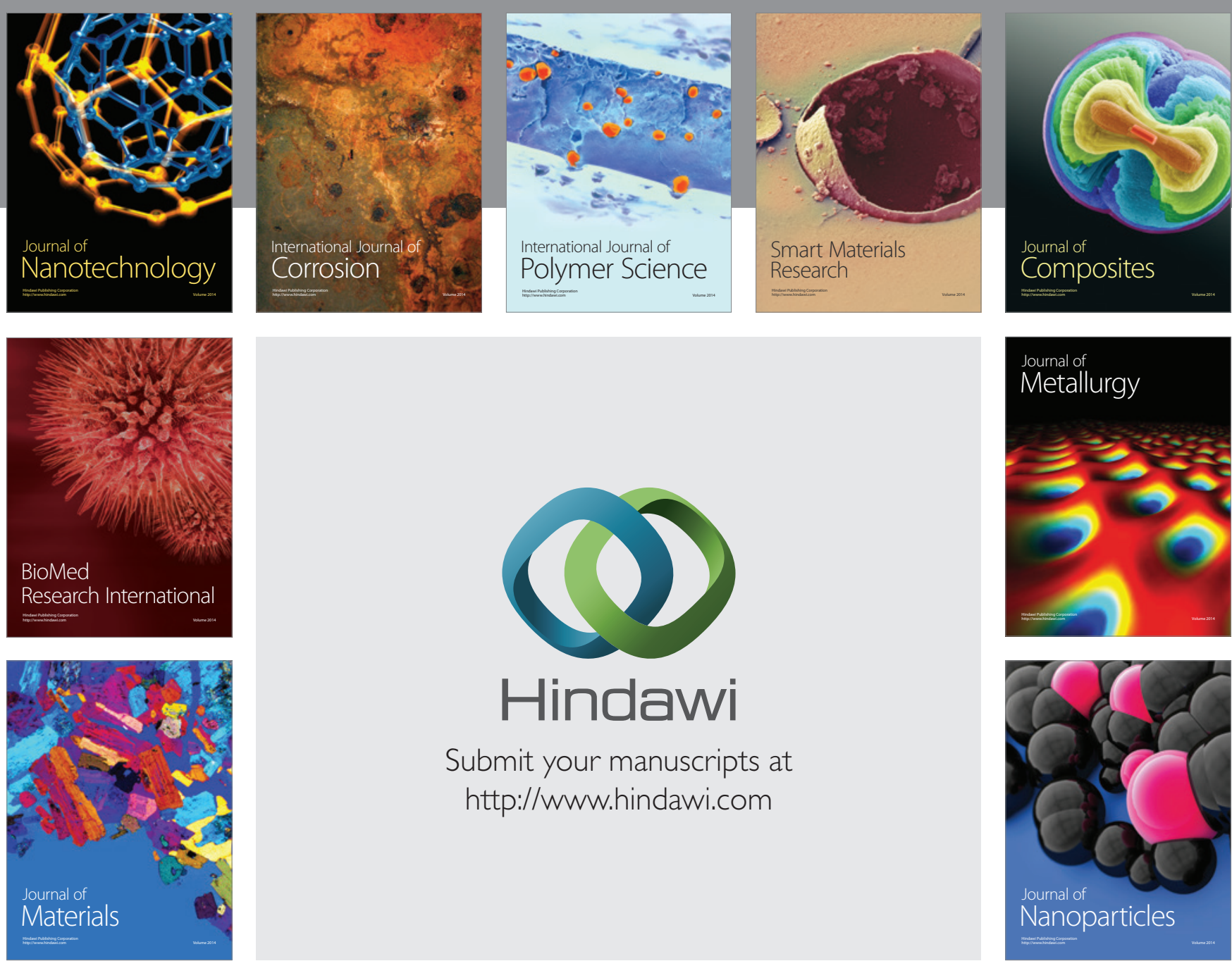

\section{Hindawi}

Submit your manuscripts at

http://www.hindawi.com

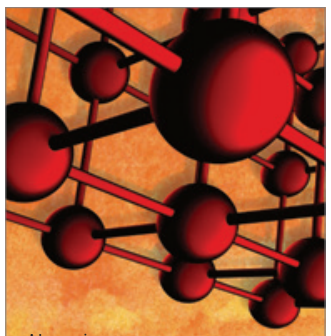

Materials Science and Engineering
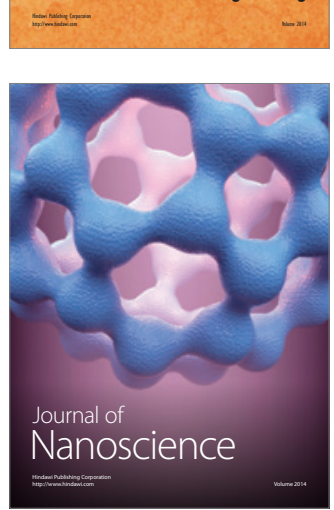
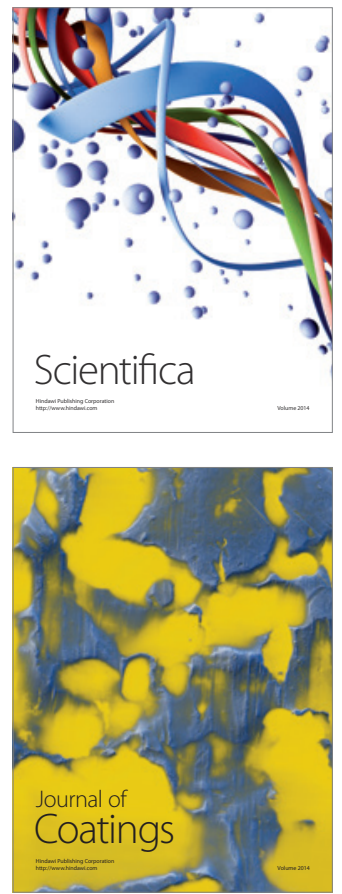
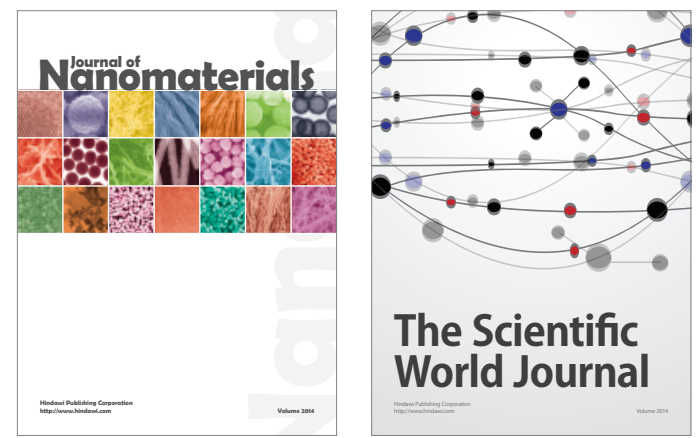

The Scientific World Journal
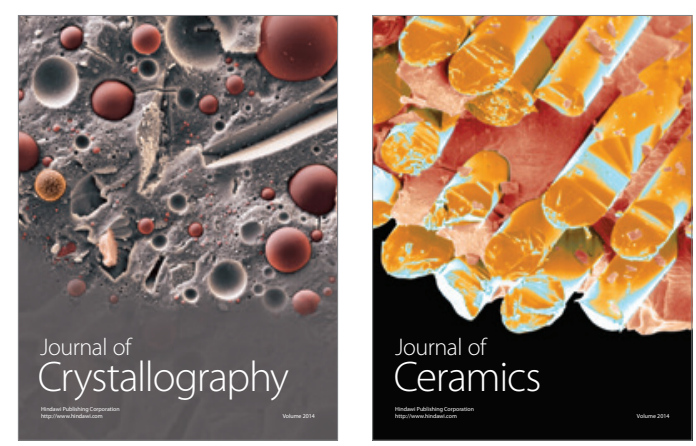
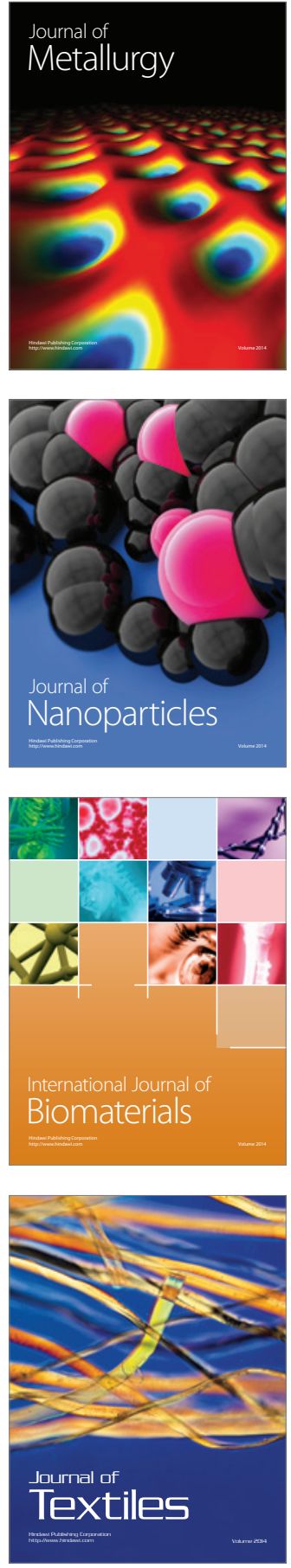